\title{
Investigation of the membrane regeneration methods by flow visualization
}

\author{
Petr Bílek ${ }^{1 *}$,Josef Brich ${ }^{1}$ \\ Technical University of Liberec, Studentská 1402/2, 461 17, Liberec 1, Czech Republic ${ }^{1}$
}

\begin{abstract}
This paper deals with an investigation of regeneration methods on a flat sheet membrane by flow visualization of the inlet side of a filter. The regeneration significantly increases performance of the membranes, extends its lifetime and saves resources. The regeneration process prevents depositing of particles on the filter surface and forming a filtration cake as well as reopens clogged pores in the filter structure. The visualization of flow helps deeper investigation of different regeneration methods because it brings a new view on the filtration/regeneration process on a thin laser sheet whilst it is recorded by a digital camera. The result is, besides the overall view, just the local concentration of the particles, which is determined by image analysis of the recorded pictures from the camera. The amount of the deposited particles on the filter is compared to the hydrodynamic data and the effect of the tangential flow and bubbling intensity parameters is investigated in the article. The tests are carried out on a low volume laboratory filtration setup - LSD 115 with a new optic filtration channel.
\end{abstract}

\section{Introduction}

Cleaning of water is a widely discussed topic because it is a part of many branches of industry. The two main mechanisms of the separation are distinguished: sedimentation and filtration. Generally, the filtration is a mechanism for separation of contaminants suspended in the medium. It operates entirely on the particle size, which is closely linked to the parameters of the filter as the pore size distribution. There are two main mechanical constructions for the water filtration: bulk filters and flat sheet filters. The bulk filters are generally used for cleaning waters with low solid content - for relatively clean waters. They are disposable and if the filter is clogged, it is impossible to clean it and use it again. On the other hand, the flat sheet filters are cleanable, because the particles create a layer called filtration cake on the surface, which can be removed easily and the filter can be regenerated and used again. There are more cleaning methods such as tangential flow (cross flow), air bubbling, backflow, pulsation, ultrasound and chemical cleaning. Finding the optimum parameters of all the cleaning methods is also very important besides the filtration parameters. It is needed to keep the filter clean and achieve the forward flow high as long as possible $[1,2]$.

This study is targeted to the tangential flow and the bubbling cleaning method and it continues with the series of measurements described in the paper [3]. In the paper [3] the experiments were carried out on the pilot filtration setup for waste water treatment, which did not allow the deeper investigation of bubbling effect and especially the effect of the tangential flow.
Because of that, the new experiments in this article were carried out on the low volume laboratory setup LSD 115 (Liquid Simulation Device) with the special optic filtration chamber made of plexiglass.

The effect of the bubbling cleaning method was recently discussed in [4]. The bubbling method is one of the unsteady-shear methods, which significantly mitigate membrane clogging. In that study, the direct observation through the membrane technique was used to evaluate the critical flux and the local parameters of bubbles. It was found out that the critical flux (the value of forward flux where the fouling starts to occur) increased with gas flow rate $[4,5]$. Also the effect of the size of the bubbles was investigated. In bioreactors it is important to have the contact area as high as possible to dissolve sufficient oxygen into the activated sludge [6]. On the other hand, the bigger size of bubbles helps more for the fouling mitigation [7]

The effect of the tangential flow was investigated in [8-10]. It is obvious that the fouling is significantly dependent on the tangential flow along the membrane surface. The shear flow can be increased by several ways, for example by a helical baffle used in [10]. The key issue of this article is to introduce the visualization method, which allows direct membrane observation in a different way than the direct observation method introduced in [4] and other studies.

\section{Experimental setup}

The scheme of the filtration setup is shown in Figure 1. The filter sample is mounted in the filtration chamber, which has an optic access on the inlet side of the filter.

\footnotetext{
* Corresponding author: petr.bilek@ $@$ tul.cz
} 
Contaminated water goes from the 5 litres beaker through the filtration chamber and comes back to the beaker. The inlet and outlet of contaminated water to the filtration chamber is divided into two channels to gain better flow uniformity. The impure water circulates to achieve the shear flow on the membrane surface. The tangential flow is controlled by the valves and a bypass and it is measured by a rotameter LB/89 (0 100 litres per hour). Also a blower is connected to the filtration chamber and it brings a fresh air to the aerator situated inside for making bubbles. Airflow is measured by an air flow meter Testo 6441. The air has to release from the filtration chamber somewhere and for this purpose a high tube is mounted to the top of the chamber. The height of the tube is given by the pressure in the upstream area. For instance, if the pressure is $10 \mathrm{kPa}$ the water level in the tube is $1 \mathrm{~m}$ high plus the height of bubbles inside the tube. Forward flow through the testing membrane is measured by digital scales PCB 10000-1.

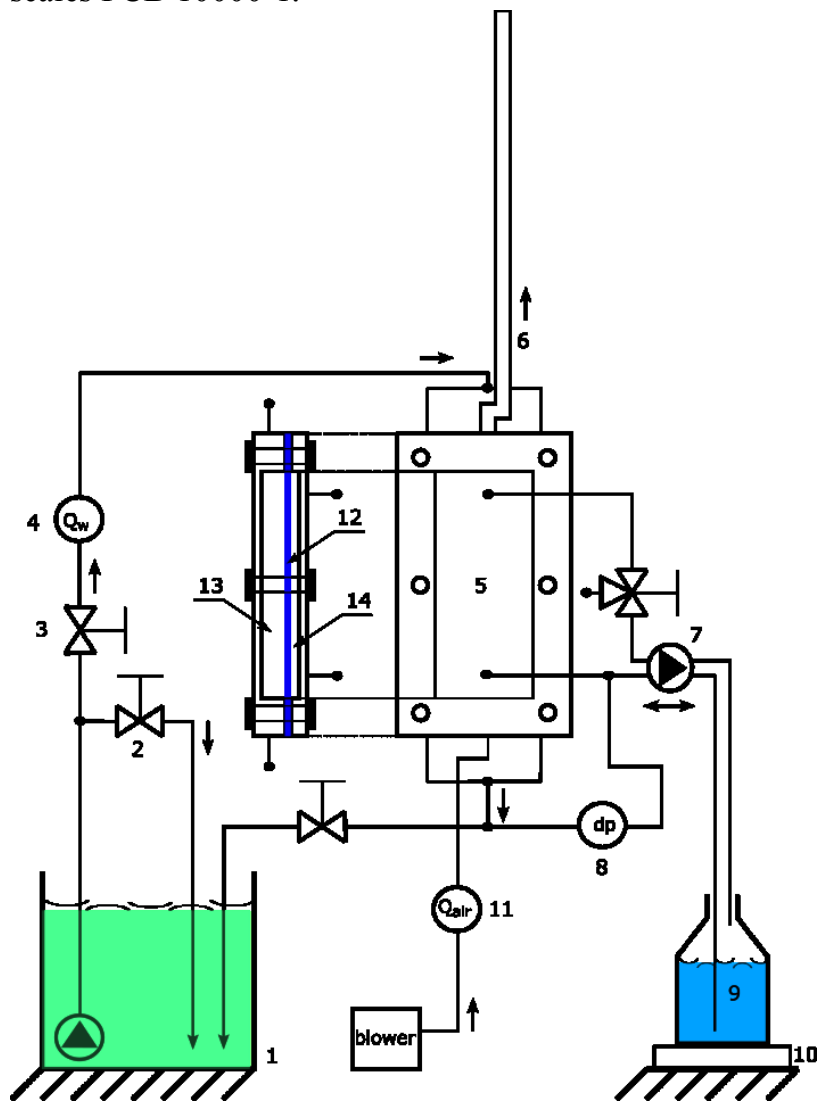

Fig. 1. Scheme of the water filtration setup LSD - 115: 1) five litre beaker for input water, 2) by-pass for setting of flowrate in the main circuit, 3) the main circuit, 4) flowmeter, 5) filtration chamber with an optic access, 6) output of the air, 7) peristaltic pump for setting higher pressure drop as well for back-flowing, 8) pressure drop transducer, 9) a jar for the clean water, 10) digital scales, 11) air flowmeter, 12) a membrane sample, 13) inlet area of the membrane sample, 14) outlet area.

The setup allows setting of the continuous cleaning methods: tangential flow and air bubbling. It is also possible to do the periodic cleaning by backflow. The setup has several sensors to measure all the basic hydrodynamic data. It is also equipped by valves for taking of water samples, following with an analysis.
In addition, the setup includes a special filtration chamber with an optic access, which allows visualizing the processes on the filter. On Figure 2, we can see the sketch of the optic filtration chamber with the laser sheet and the digital camera.

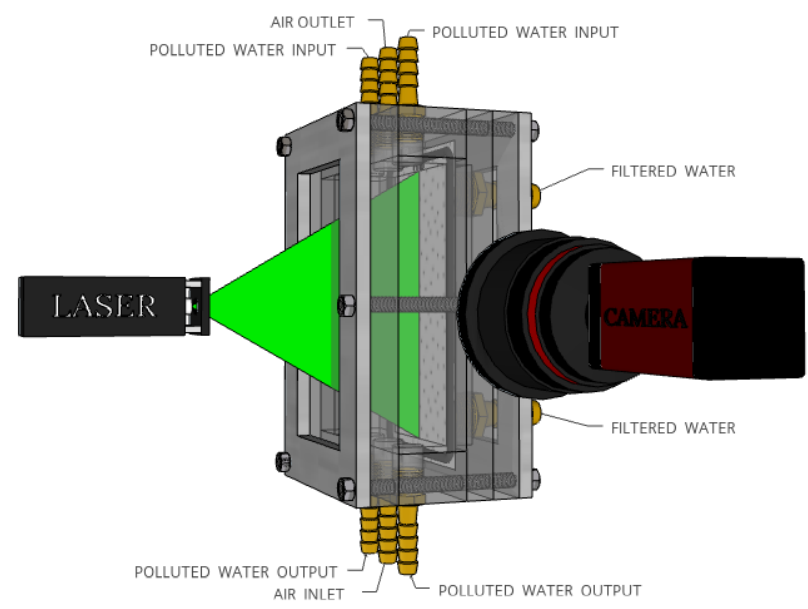

Fig. 2. Detail sketch of the filtration cell allowing a view on the membrane. We can see also the laser unit and the camera in the right angle position.

The laser sheet is used for illuminating seeding particles present in water and the digital camera captures the scene and makes images. The hardware configuration is similar to the PIV method [11]. The laser sheet is generated by Powell lens $\left(30^{\circ}\right)$ and a continuous LED laser module $(532 \mathrm{~nm}, 121 \mathrm{~mW})$. The digital camera is made by Allied Vision, the type is Pike $\mathrm{F}-210 \mathrm{~B} / \mathrm{C}$, the key parameters are: $50 \mathrm{fps}, 1920 \times 1080 \mathrm{px}, 16$ bit depth, equipped by the lens (Samyang $35 \mathrm{~mm}, \mathrm{~F} 1.4$ ). The camera is controlled in the software AVT Smart View.

\section{Materials and methods}

The nanofibrous filter is made of three layers. The first and the most important electrospun nanoporous layer is made of polyamide 6 and it is bound with the meltblown support layer made of polyester by a sparse mesh also made of polyamide 6 . The dimension of the active area of each filter sample is $50 \times 95 \mathrm{~mm}$, so the resulting active area is $0.00475 \mathrm{~m}^{2}$. The mean pore size is around $0.5 \mu \mathrm{m}$ and the maximum pore size is around $1.5 \mu \mathrm{m}$ (measured by a bubble point test). Air permeability is $7-10 \mathrm{~m} / \mathrm{s} \cdot \mathrm{Pa}$ and the basis weight of the nanolayer is around $3 \mathrm{~g} / \mathrm{m}^{2}$. The thickness of the whole membrane is around $160 \mu \mathrm{m}$.

The filter samples were tested by distilled water with artificial seeding particles from the company Bang laboratories, Inc. The size of the monodisperse testing particles was chosen on $0.5 \mu \mathrm{m}$ to maximizing visibility of the filtration cake. The mean pore size of the membrane is close to the size of the particles. The particles are made of polystyrene, they are spherical and the density is $1.05 \mathrm{~g} / \mathrm{ml}$ which is comparable with the density of distilled water: $0.998 \mathrm{mg} / \mathrm{ml}\left(20{ }^{\circ} \mathrm{C}\right)$. They have good optical properties as well (refractive index for polystyrene is 1.595 , for water 1.334 ). The concentration of the particles in distilled water was $5 \mathrm{mg} /$ litre. 


\subsection{The measuring method}

In this article we are focused on the input parameters: the tangential velocity $v_{t}$ and the intensity of bubbling given by air flow rate $Q_{a i r}$. The velocity of water with the particles around the filter is calculated from the tangential flow $Q_{w}[\mathrm{LPH}]$ according to

$$
v_{t}=\frac{Q_{w}}{S} \cdot \frac{1}{3600}\left[\frac{m m}{s}\right] \text {. }
$$

The square area, where water flows along the membrane, is $S=25 \times 50 \mathrm{~mm}=0.00125 \mathrm{~m}^{2}$. The pressure drop is kept on the constant level, $\Delta \mathrm{p}=20 \mathrm{kPa}(8+12 \mathrm{kPa})$. The reverse pressure drop is also always the same $-20 \mathrm{kPa}$ and always $150 \mathrm{ml}$ of distilled water was pumped back during the backflow. The concentration of solid impurities is determined by a laboratory turbid meter Lovibond TB 300 IR. We assume the mass concentration [ug/l] is proportional to the turbidity [NTU] (the particles are monodisperse). The filtration efficiency is calculated according to

$$
E F=\left(1-\frac{C_{2}}{C_{1}}\right) \cdot 100[\%],
$$

where $C_{1}[\mathrm{ug} / \mathrm{l}]$ is inlet concentration and $C_{2}$ outlet. We are looking for the best cleaning conditions to keep the filter surface clean as much as possible. The information is generally obtained from the hydrodynamic values. The most important is the forward flow rate. Forward flow through the membrane directly points out on the efficiency of the cleaning method. As high the forwards flow is as efficient the cleaning method is. The new on this measurement is that it is possible to verify the hydrodynamic data by the visualization. This technique confirms the correctness of the generally available data and makes the measurement more robust.

During the backflow, the particles stacked on the filter surface are released again to the water and the filter is washed, Figure 3. If the backwash intensity and the other parameters are always the same, the quantity of the particles released from the filter surface points out on the efficiency of the continuous cleaning method. The efficiency of the continuous cleaning method was as low as more particles stacked on the filter. It literally means that the particles were not washed properly during the continuous cleaning (by tangential flow or bubbling) and created a filtration cake, which is then washed by the backflow. The visualization method is able to determine amount of released particles during the backwash and shows which parameters of the tangential flow and bubbling intensity are the best.

A new sample of the filter and also the new solution of testing water were used for every test. It is very important to keep the same conditions for every test. The membrane is not stable during the filtration because of the irreversible clogging and bacteria growth. Also the particles in the water are making clusters and stack on the walls in time. Every experiment took 20 minutes.

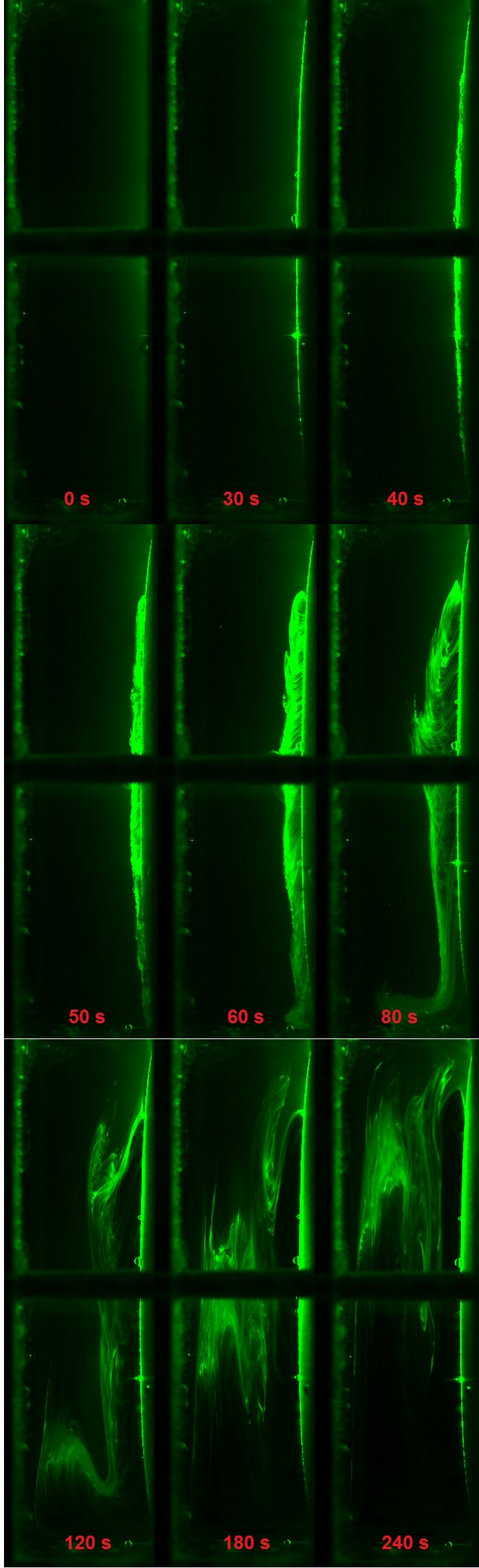

Fig. 3. Images of the inlet area of the membrane taken in different times during cleaning by back flow (particles: $0.5 \mu \mathrm{m}$ ). 


\section{Results and discussion}

\subsection{Effect of the particle size}

The different particles were used to find the optimal size (diameter) for the experiments. There are more aspects lying on this issue. The particles have to be visible enough in the laser light and also they should simulate the main pollution in the real water. After the consideration of all the aspects and also the experiments with different sizes, the $0.5 \mu \mathrm{m}$ particles were chosen to be the most suitable and they only were used furthermore.

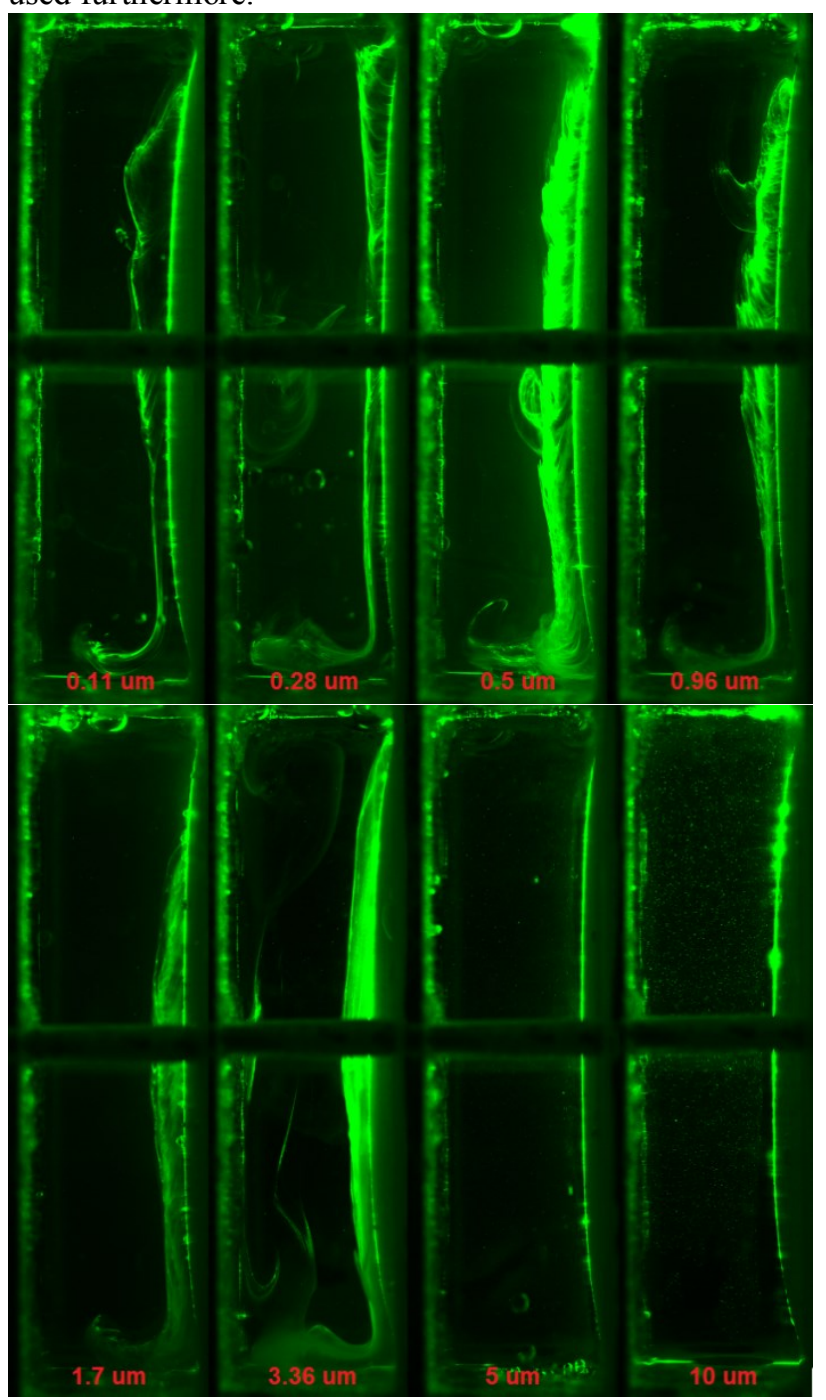

Fig. 4. Images of the inlet area of the membrane taken for different particle sizes approximately at the same time.

The shiniest filtration cake was in case of $0.5 \mu \mathrm{m}$ particles, Figure 4. From the size $0.11 \mu \mathrm{m}$ to $1.7 \mu \mathrm{m}$ the mass concentration of the particles was the same $5 \mathrm{mg} /$ litre. The concentration for $3.36 \mu \mathrm{m}$ was higher around $15 \mathrm{mg} /$ litre. For the last two sizes the concentration was $20 \mathrm{mg} /$ litre. The hydrodynamic data are summarized in Table 1 and shown in graphs in Figure 5. The tangential flowrate was zero as well as the air flowrate. The filtration efficiency was calculated according to (1). The amount of filtered water after 20 minutes of filtration is increasing with the size of particles from range $0.11-3.36 \mu \mathrm{m}$. For sizes 5 and 10 $\mu \mathrm{m}$ (it was measured 2 times to exclude errors) the flowrate significantly decreases with the particle size, which points out on the clogging. On the other hand, no filtration cake is seen on the images for this size of particles. This contradiction can be caused by a very fluffy filtration cake. Also the mass concentration was too low for such big particles to show something in the images. The irreversible clogging can play some role as well. The bigger sizes of the testing particles ( 5 and 10 $\mu \mathrm{m})$ are neither monodisperse nor spherical. They are round and the size distribution is $\pm 50 \%$ of their mean size.

Tab. 1. Results from the experiments with different sizes of testing particles.

\begin{tabular}{|c|c|c|c|c|}
\hline $\begin{array}{c}\text { Size of } \\
\text { particles } \\
{[\mu \mathrm{m}]}\end{array}$ & $\begin{array}{c}\text { Turbidity of } \\
\text { input water } \\
{[\mathrm{NTU}]}\end{array}$ & $\begin{array}{c}\text { Turbidity of } \\
\text { output } \\
\text { water [NTU] }\end{array}$ & $\begin{array}{c}\text { Filtration } \\
\text { efficiency } \\
{[\%]}\end{array}$ & $\begin{array}{c}\text { Filtered } \\
\text { water after } \\
20 \text { min. [g] }\end{array}$ \\
\hline 0.11 & 5.45 & 0.08 & 98.5 & 510 \\
\hline 0.28 & 42.6 & 0.07 & 99.8 & 1075.9 \\
\hline 0.5 & 36.3 & 0.18 & 99.5 & 1198.1 \\
\hline 0.96 & 22.2 & 0.46 & 97.9 & 1171.2 \\
\hline 1.7 & 21.5 & 0.08 & 99.6 & 1670.5 \\
\hline 3.36 & 15.4 & 1.29 & 91.6 & 1757.2 \\
\hline 5 & 11.7 & 0.76 & 93.5 & 1159.6 \\
\hline 10 & 16.9 & 0.18 & 98.9 & 653.6 \\
\hline
\end{tabular}

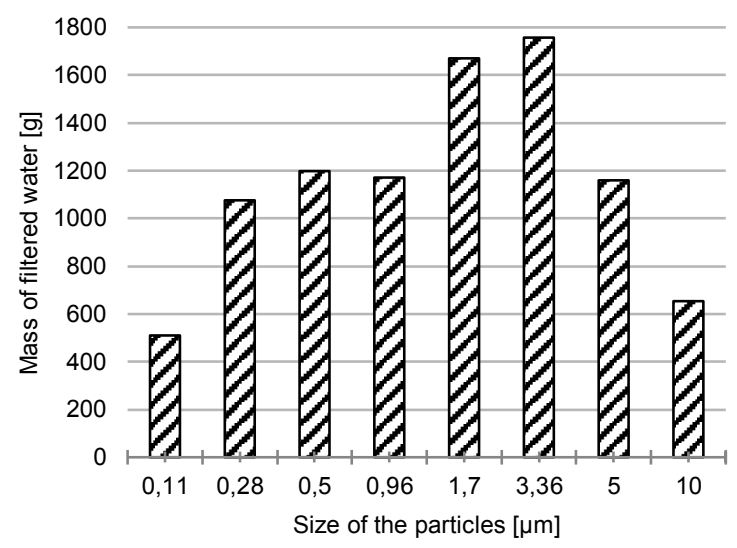

Turbidity of input water $\square$ Turbidity of output water •Filtration efficiency

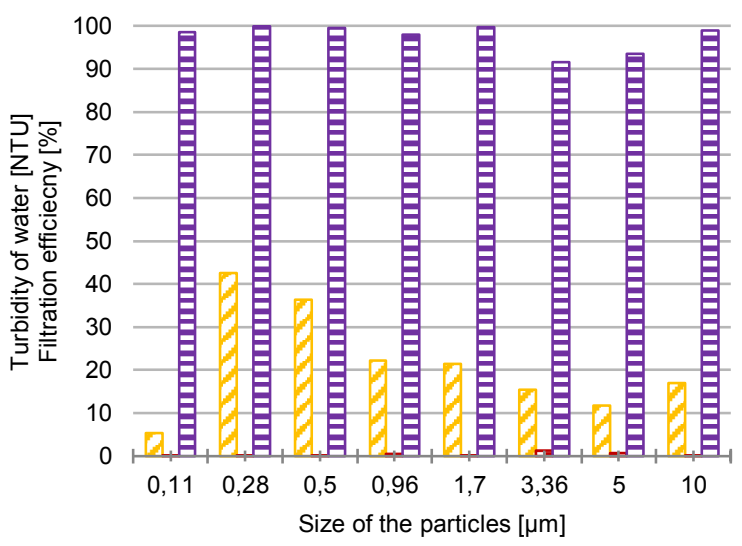

Fig. 5. Graphs of forward flowrate, turbidity and filtration efficiency for different sizes of the testing particles. 


\subsection{Image analysis}

The image analysis was carried out in ImageJ [12] and the following data were processed in MS Excel 2010. Four different sized evaluative areas are shown on Figure 6. The mean digital grey value was calculated for every image from the beginning to the end of backflow. It was around 300 images -5 minutes for every experiment. From the series of mean values in time, the highest one was always chosen for each experiment and for each evaluative area. Camera settings were: lens F2.8, shutter time $2 \mathrm{~ms}$ and gain 100.

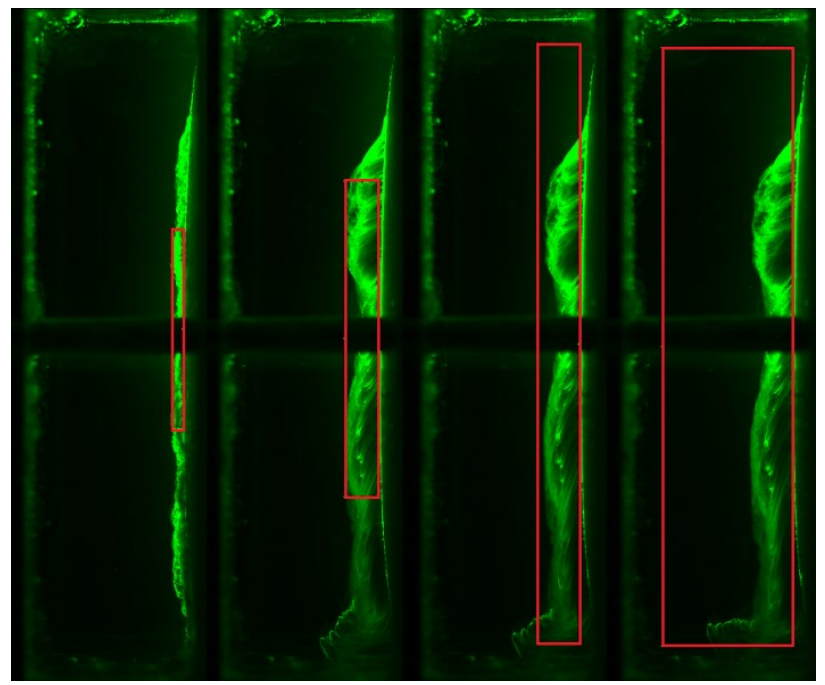

Fig. 6. Different evaluation areas: tiny, small, medium and the big evaluative area.

The tangential flow was set gradually from 0 to 100 litres per hour with the step of 25 litres per hour (LPH). The air flow rate was always zero during this measurement. Then the air flow rate was changed from 0 to 1 and then to 10 litres per minute during the zero tangential flow. Table 2 summarizes all the experiments and the input parameters.

Tab. 2. Settings of the input parameters.

\begin{tabular}{|c|c|c|c|}
\hline $\begin{array}{c}\text { Test } \\
\text { number }\end{array}$ & $\begin{array}{c}\text { Tangential } \\
\text { flowrate } \\
{[\mathrm{LPH}]}\end{array}$ & $\begin{array}{c}\text { Tangential } \\
\text { velocity } \\
{[\mathrm{mm} / \mathrm{s}]}\end{array}$ & $\begin{array}{c}\text { Air } \\
\text { flowrate } \\
{[\mathrm{LPM}]}\end{array}$ \\
\hline 1 & 0 & 0.0 & 0 \\
\hline 2 & 0 & 0.0 & 1 \\
\hline 3 & 0 & 0.0 & 10 \\
\hline 4 & 25 & 5.6 & 0 \\
\hline 5 & 50 & 11.1 & 0 \\
\hline 6 & 75 & 16.7 & 0 \\
\hline 7 & 100 & 22.2 & 0 \\
\hline
\end{tabular}

Table 3 shows the results from the image analysis. We can see that the mean digital grey value decreases with the increasing size of evaluative area, because more black pixels are included to the calculation. If the filtration cake is not stable, we can get error values for tiny evaluative area (Test $4-$ small and medium evaluative area in Table 3). Also the results in time (it is not seen in Table 3) fluctuate more for tiny evaluative area than for big one. It is obvious that the big evaluative area, which covers all the inlet area of the filter, gives us more accurate and reliable results. The results for the big evaluative area were used in the following data processing.

Tab. 3. Results from the image analysis.

\begin{tabular}{|c|c|c|c|c|}
\hline \multirow{2}{*}{$\begin{array}{c}\text { Test } \\
\text { number }\end{array}$} & \multicolumn{4}{|c|}{ Mean digital grey value in evaluative areas [-] } \\
\cline { 2 - 5 } & Tiny & Small & Medium & Big \\
\hline 1 & 78.63 & 45.05 & 31.01 & $\mathbf{1 5 . 4 4}$ \\
\hline 2 & 59.8 & 33.85 & 25.6 & $\mathbf{1 4 . 7 2}$ \\
\hline 3 & 30.92 & 24.78 & 21.62 & $\mathbf{1 3 . 8 7}$ \\
\hline 4 & 78.4 & 40.02 & 26.29 & $\mathbf{1 5 . 0 4}$ \\
\hline 5 & 76.26 & 44.1 & 30.14 & $\mathbf{1 4 . 4 4}$ \\
\hline 6 & 75.09 & 42.35 & 29.51 & $\mathbf{1 3 . 8 4}$ \\
\hline 7 & 74.54 & 33.48 & 25.86 & $\mathbf{1 2 . 9}$ \\
\hline
\end{tabular}

\subsection{Effect of the tangential flow and the bubbling intensity}

The images for different tangential flow rates are not presented here, because they are not so much interesting. It is hard to distinguish them from each other by a naked eye. The bubbling intensity versus air flowrate during air bubbling is seen in Figure 7. The pictures for the different bubbling intensities during backflow are shown in Figure 7. For the 1 LPM of air flowrate the shape of the filtration cake looks very similar to the other experiments (for ex. Figure 3). In case of very high bubbling intensity (air flowrate 10 LPM) the shape of the filtration cake at the same time is the same too. But in few tens of seconds later, there the second filtration cake appeared, which was very non-uniform, literally torn apart. It is obvious that the bubbles pressed the filtration cake on the membrane surface by its movements. The filtration cake is denser and it needs more time to be slushed off.

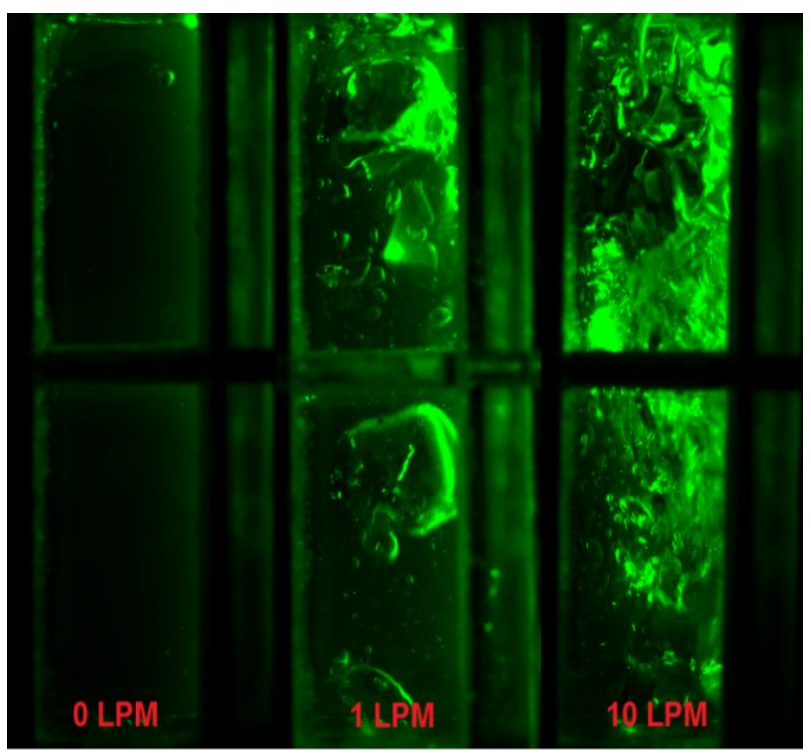

Fig. 7. Images of the inlet area of the membrane sample taken during zero air flow, 1 litre per minute and 10 litres per minute. 


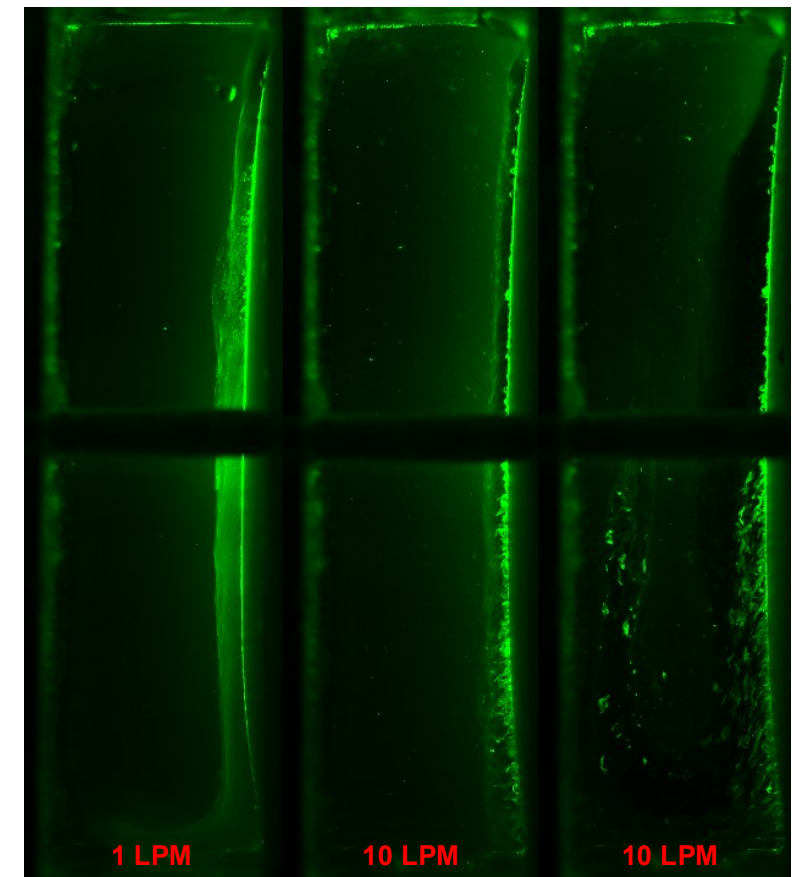

Fig. 8. Images of the inlet side of the membrane during backflow for two bubbling intensities.

Tab. 4. Results from the measurements

\begin{tabular}{|c|c|c|c|c|c|}
\hline \multirow[t]{2}{*}{$\begin{array}{c}\text { Test } \\
\text { number }\end{array}$} & \multirow{2}{*}{$\begin{array}{c}\text { The first } \\
\text { minute of } \\
\text { filtration } \\
\text { [g] }\end{array}$} & \multicolumn{2}{|c|}{$\begin{array}{l}\text { Filtered water before } \\
\text { backflow, } 20 \text { minutes } \\
\text { of filtration [g] }\end{array}$} & \multicolumn{2}{|c|}{$\begin{array}{l}\text { Filtered water after } \\
\text { backflow, } 1 \text { minute of } \\
\text { filtration [g] }\end{array}$} \\
\hline & & Measured & Corrected & Measured & Corrected \\
\hline 1 & 130 & 1393 & 796 & 75 & 43 \\
\hline 2 & 78 & 418 & 477 & 8.9 & 10 \\
\hline 3 & 69 & 419 & 521 & 21 & 26 \\
\hline 4 & 91 & 894 & 894 & 51 & 51 \\
\hline 5 & 112 & 1214 & 934 & 50 & 38 \\
\hline 6 & 106 & 1083 & 904 & 51 & 43 \\
\hline 7 & 100 & 1231 & 1109 & 56 & 50 \\
\hline
\end{tabular}

The hydrodynamic results are summarized in Table 4. We can see that the amount of filtered water in the first minute is very fluctuating for the tests from 1 to 7 although the membrane samples were cut from the same membrane sheet. Every sample of the membrane should be the same, but it is obvious it is not. The values of forward flowrate had to be corrected percentages according to the flowrate in the first minute.

The final graphs demonstrating the correlation between the hydrodynamic data and data from the visualization are shown in Figures 9 and 10. We can see that the mean digital grey value decreases with increasing tangential flow, which points out on fewer particles presented in the filtration cake. The forward flowrate on the contrary increases. This correlates with the theory, that the tangential flow mitigates the membrane fouling. The forward flowrate was measured also after backflush to see how the filter was regenerated. The values are fluctuating without any trend.

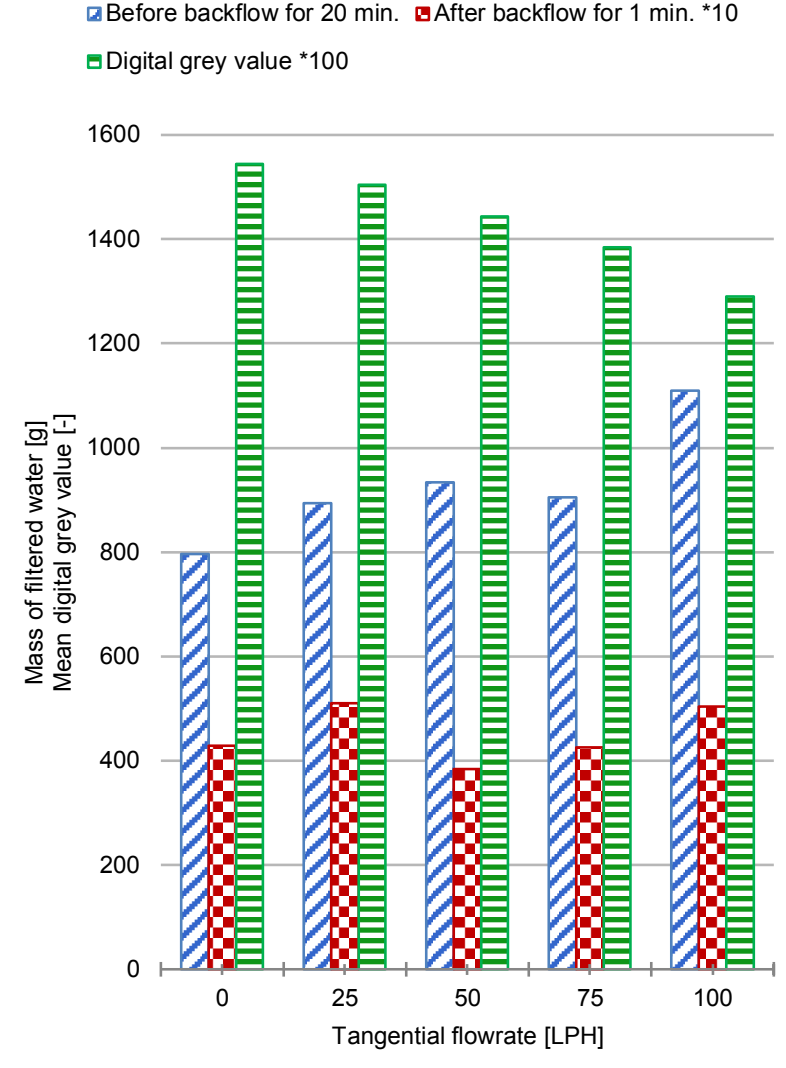

Fig. 9. Graph comparing the forward flow rate and the results from image analysis for tangential flow.

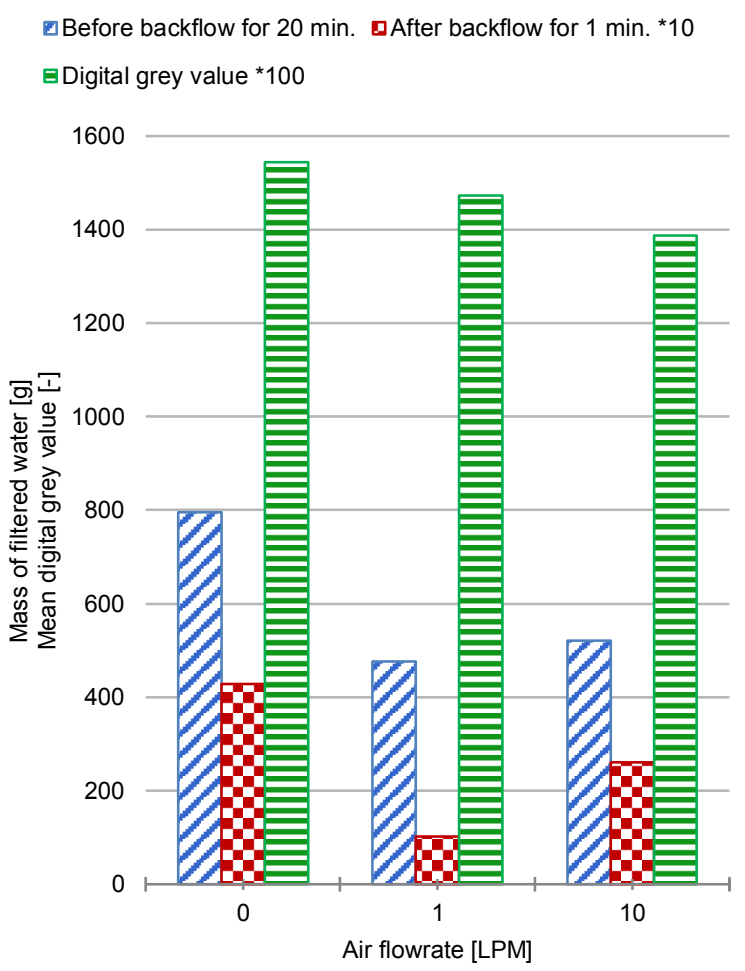

Fig. 10. Graph comparing the forward flow rate and the results from image analysis for bubbling intensity.

Filtration efficiency was around $99 \%$ for all the experiments proving that filters were not damaged and there was no water bypassing. The effect of bubbles is very significant. When the bubbles occurred the forward 
flowrate very significantly dropped. The bubbles presented in the water influence the filtration process. The positive effect of bubbling is not possible to proof from the hydrodynamic data during short term measurement. On the other hand, according to the digital grey value the filtration cake was ticker in case of higher intensities of bubbling. Intensive bubbling decreases lifetime of a filter, because bubbles scratch the filter surface.

\section{Conclusion}

The cleaning of the filters is a very important part of the filtration process. Optimal parameters of the continuous cleaning methods as the tangential velocity of water along the filter surface and the intensity of air bubbling were investigated in this paper. Besides the usual hydrodynamic data also the new information was achieved from the visualization system, which is the major issue of the paper. The visualization system is composed from the continuous laser sheet and the digital CCD camera. The new optic filtration cell was made of plexiglass for this purpose. After the image analysis of recorded images, we got the information about amount of particles, which were removed from the inlet side during backflush. The image analysis is the most critical issue of the measuring chain. The most important parameters are the size and position of an evaluative area, time, pressure drop and flowrate.

The results summarized in Figure 9 and Figure 10 show that the values obtained from the visualization method correlate with the hydrodynamic data. As high the tangential flow is as cleaner filter surface is. The same applies for the bubbling intensity. We can see that the hydrodynamic data are not always satisfactory in the short term measurement. On the other hand the data from the image analysis fits completely with the theory even in short term measurement. It is the most promising property of the visualization method - it is not needed to run a test too long to find out the best cleaning parameters. In addition, we know more about the system only from the observing. For instance, we can see the different shape of the filtration cake versus bubbling intensity and better understand the filtration and the cleaning process.

It is obvious that the method can be improved rapidly by using fluorescent particles to distinguish all the reflections from the light emitted by the particles. Interesting will be the effect of the tangential flow on the filtration cake for higher values than $100 \mathrm{LPH}$. Also the bubbling intensity can be investigated with a softer step to see the differences. The error bars are not presented in the results. The main purpose of the paper is to show possibilities of this direct observing method on the first results, proving helpfulness during the investigation of the cleaning processes.

Generally, the low volume filtration setup LSD 115 is less stable compared to the big pilot filtration setup used in [3]. On the other hand, the preparation of the filter samples and running the tests is significantly less timeconsuming and suits more for this purpose.
The results of this project LO1201 were obtained through the financial support of the Ministry of Education, Youth and Sports in the framework of the targeted support of the "National Programme for Sustainability I".

\section{References}

1. Spellman F R, Handbook of water and wastewater treatment plant operations, CRC Press 2009, p 826, ISBN: 13-978-1-4200-7530-4

2. Sparks T, Chase G, Section 4 - Solid-Liquid Filtration - Filters and Filtration Handbook (Sixth Edition), Butterworth-Heinemann 2016, pp. 199295, ISBN 9780080993966

3. Bílek, P., Hrůza, J. (2018). Optimization of the cleaning process on a pilot filtration setup for waste water treatment accompanied by flow visualization. Paper presented at the EPJ Web of Conferences, 180, p 5

4. Wang, J., Fane, A. G., Chew, J. W. Effect of bubble characteristics on critical flux in the microfiltration of particulate foulants (2017) Journal of Membrane Science, 535, pp. 279-293.

5. Qaisrani, T. M., Samhaber, W. M. Impact of gas bubbling and backflushing on fouling control and membrane cleaning (2011) Desalination, 266 (1-3), pp. 154-161

6. Wastewater treatment: Bubbling up for major energy savings (2011), Filtration + Separation, 48 (4), pp. 42-43

7. Li, Q.Y., Cui, Z.F., Pepper, D.S. Effect of bubble size and frequency on the permeate flux of gas sparged ultrafiltration with tubular membranes (1997) Chemical Engineering Journal, 67 (1), pp. 71-75

8. Choi, H., Zhang, K., Dionysiou, D. D., Oerther, D. B., Sorial, G. A. Effect of permeate flux and tangential flow on membrane fouling for wastewater treatment (2005) Separation and Purification Technology, 45 (1), pp. 68-78

9. Knutsen, J. S., Davis, R. H. (2006). Deposition of foulant particles during tangential flow filtration, Journal of Membrane Science, 271 (1-2), pp. 101113

10. Horie, T., Shiota, S., Akagi, T., Ohmura, N., Wang, S., Eze, V., Hirata, Y. (2018). Intensification of hollow fiber membrane cross-flow filtration by the combination of helical baffle and oscillatory flow, Journal of Membrane Science, 554, pp. 134-139

11. Tropea C, Yarin A, Foss J F, Springer Handbook of Experimental Fluid Mechanics 2007, p 1557, ISBN: 978-3-662-49162-1

12. ImageJ. Image analysis software [online: 22.9.2018], URL:<http://rsbweb.nih.gov/ij/index.html>. 